\title{
Performance of Multicarrier DS-SS with Imperfect Carrier Phase on Multipath Rayleigh Fading Channels
}

\author{
K. R. Shankar kumar and A. Chockalingam \\ Department of Electrical Communication Engineering \\ Indian Institute of Science, Bangalore 560012, INDIA
}

\begin{abstract}
In this paper, we analyze the bit error performance of multicarrier direct-sequence spread spectrum (DS-SS) systems with imperfect carrier phase on multipath Rayleigh fading channels. We consider the phase errors arising from the receiver phase locked loops (PLL's) on the individual sub-carriers to be Tikhonov distributed. We derive a simple upper bound on the average bit error probability by approximating the phase error loss function by a staircase function. We show that the bound is inexpensive to compute and is reasonably accurate. We present the effect of imperfect phase coherence on the bit error performance under various channel and system conditions.

Keywords - Multicarrier systems, DS-SS, imperfect carrier phase, Tikhonov distribution.
\end{abstract}

\section{Introduction}

Multicarrier (MC) direct-sequence code division multiple access (DS-CDMA) is an effective approach to combat fading and various kinds of interference [1],[2],[3]. In [2], Kondo and Milstein proposed a multicarrier DS-CDMA scheme where a data sequence multiplied by a spreading sequence modulates disjoint multiple carriers. The receiver provides a correlator for each carrier and the outputs of the correlators are combined with a maximal-ratio combiner. Bandlimited spreading waveforms are used to prevent self-interference and the system performance is evaluated assuming perfect phase coherence in a slowly fading Rayleigh channel, with all sub-bands being subjected to independent fading.

Our contribution in this paper is the analysis of the bit error performance of a multicarrier DS-SS system, when the phase estimates of the carrier are imperfect. In [6], Viterbi has shown that the phase error in a first-order PLL follows a Tikhonov distribution. In [5], Eng and Milstein have analyzed the performance of a partially coherent single carrier DS-SS system. In this paper, we provide the performance analysis of a multicarrier DS-SS system

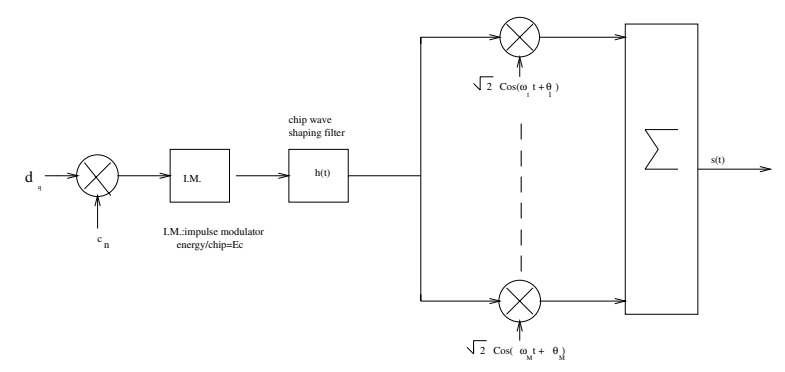

Figure 1: Multicarrier DS-SS Transmitter

with imperfect carrier phase on multipath Rayleigh fading channels. A key step in our analysis is the approximation of the phase error loss function by a staircase function.

The rest of the paper is organized as follows. In Section 2, we present the system model including multicarrier DS-SS transmitter, fading channel model and the receiver with imperfect carrier phase. In Section 3, we present the preformance analysis including the derivation of an upper bound on the bit error probability. Numerical results and discussions are presented in Section 4. Conclusions are presented in Section 5.

\section{System Model}

Consider a multicarrier system where the available bandwidth $W$ is divided into $M$ equal-width, disjoint frequency bands, such that the bandwidth of each sub-band is given by $W_{i}=W / M, i=1,2, \ldots, M$. Each subband carries a narrowband DS-SS waveform of bandwidth given by $W_{i}=(1+a) / M T_{c}, i=1,2, \ldots, M$, where $0<a \leq 1$ is the measure of excess bandwith of the system, and $M T_{c}$ is the chip duration of the multicarrier DSSS system.

Fig. 1 shows the transmit chain of the multicarrier DSSS system considered. The binary sequence representing the data is denoted by $d_{q}$, and the pseudorandom spreading sequence is denoted by $c_{n}$. Assume that there are $N$ chips per data symbol. The sequence $d_{q} c_{n}$ modulates an impulse train where the energy per chip is $E_{c}$. The chip 


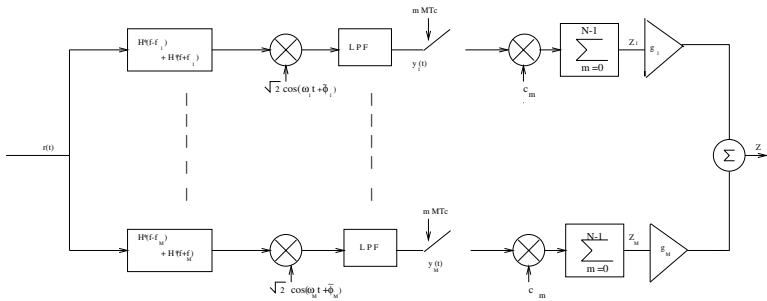

Figure 2: Multicarrier DS-SS Receiver with imperfect carrier phase

wave shaped signal then modulates multiple sub-carriers with frequencies $\omega_{1}, \omega_{2}, \ldots, \omega_{M}$ and phases $\theta_{1}, \theta_{2}, \ldots, \theta_{M}$. The transmitted signal $s(t)$ is given by

$s(t)=\sqrt{2 E_{c}} \sum_{n=-\infty}^{\infty} d_{q} c_{n} h\left(t-n M T_{c}\right) \sum_{i=1}^{M} \cos \left(\omega_{i} t+\theta_{i}\right)$,

where $q=\lfloor n / N\rfloor, h(t)$ is the impulse response of the chip wave shaping filter, and $\theta_{i}$ is the $i^{t h}$ sub-carrier's random phase uniformly distributed over $[0,2 \pi]$.

As in [2], we assume that the multipath fading on individual sub-carriers is frequency non-selective and that all sub-bands are subject to independent fading ${ }^{1}$. Consequently, the channel transfer function of $i^{t h}$ sub-band can be characterized by $\alpha_{i} e^{j \phi_{i}}$, where $\alpha_{i}$ represents the fade amplitude on the $i^{t h}$ sub-carrier which is a Rayleigh random variable with $E\left[\alpha_{i}^{2}\right]=1$, and $\phi_{i}$ is the channel introduced random phase on the $i^{\text {th }}$ sub-carrier which is a uniform random variable over $[0,2 \pi]$. The received signal $r(t)$ is then given by

$$
\begin{array}{r}
r(t)=\sqrt{2 E_{c}} \sum_{n=-\infty}^{\infty} d_{q} c_{n} h\left(t-n M T_{c}-\tau\right) \\
\cdot \sum_{i=1}^{M} \alpha_{i} \cos \left(\omega_{i} t+\theta_{i}^{\prime}\right)+n(t),
\end{array}
$$

where $\theta_{i}^{\prime}=\theta_{i}+\phi_{i}$ and $n(t)$ is the AWGN with a psd of $\eta_{0} / 2$.

Fig. 2 shows the multicarrier DS-SS receiver with imperfect carrier phase. We assume that the chip matched filter satisfies the Nyquist criterion to guarantee that the DS waveforms on individual sub-carriers do not overlap. With $H(f)$, the Fourier transform of the chip wave shaping filter impulse response $h(t)$, we define $x(t) \equiv$ $F^{-1}|H(f)|^{2}$, and assume $\int_{-\infty}^{\infty}|H(f)|^{2} d f=1$. In the performance analyses in [2],[4], perfect knowledge of the phases of all sub-carriers has been assumed. However, the locally generated sub-carriers typically contain random phase errors arising from the PLLs used to track the individual sub-carriers. Accordingly, we assume that the

\footnotetext{
${ }^{1}$ These assumptions can be valid if we choose $M, a$ and $T_{c}$ such that $\frac{1}{B_{c} T_{c}} \leq M \leq(1+a) \frac{1}{B_{c} T_{c}}$, where $B_{c}$ is the coherence bandwidth of the channel [2]. The effect of non-independent fading (i.e., correlated fading) among various sub-carriers, assuming perfect coherence, is analyzed in [4].
}

locally generated sub-carriers have the phases

$$
\tilde{\theta}_{i}=\theta_{i}^{\prime}+\triangle_{i}, \quad i=1,2, \ldots, M,
$$

where $\triangle_{i}$ is the phase error in the $i^{t h}$ sub-carrier's PLL.

It has been shown that when the PLLs are in lock condition, the phase errors follow Tikhonov distribution [6]. The phase error $\triangle_{i}$ has the Tikhonov pdf given by

$$
p\left(\triangle_{i}\right)=\frac{e^{\gamma_{i} \cos \left(\triangle_{i}\right)}}{2 \pi I_{0}\left(\gamma_{i}\right)}, \quad-\pi<\triangle_{i}<\pi .
$$

In the above, $I_{0}($.$) is the zeroth order modified Bessel$ function of the first kind, and $\left\{\gamma_{i}\right\}$ are the instantaneous loop SNRs which are themselves random variables with exponential distributions because $\gamma_{i}$ is proportional to $\alpha_{i}^{2}$ [7]. The pdf of $\gamma_{i}$ is given by

$$
p\left(\gamma_{i}\right)=\frac{1}{\nu_{i}} e^{\frac{-\gamma_{i}}{\nu_{i}}},
$$

where $\nu_{i}=E\left[\gamma_{i}\right]$ is the average loop SNR. We assume that the average loop SNR is the same in all the $M$ loops, i.e., $\nu_{1}=\nu_{2}=\ldots=\nu_{M}=\nu$. In order not to invalidate the use of Tikhonov distribution for phase errors, we assume that the fade bandwidth is smaller than the loop bandwidths of the PLLs so that $\gamma_{i}$ may be treated as being constant over several bit intervals.

\section{Performance Analysis}

Assuming perfect code and bit timing, but imperfect carrier phases at the receiver, the output of the chip matched filter in the $i^{t h}$ branch, $y_{i}(t)$, can be written as (ignoring double frequency term after low pass filtering)

$$
y_{i}(t)=S_{y i}(t)+N_{y i}(t),
$$

where the signal component $S_{y i}(t)$ can be written as

$$
S_{y i}(t)=\alpha_{i} \sqrt{E_{c}} \cos \left(\triangle_{i}\right) \sum_{n=-\infty}^{\infty} d_{q} c_{n} x\left(t-n M T_{c}\right),
$$

and the noise component $N_{y i}(t)$ can be written as

$$
N_{y i}(t)=\operatorname{LPF}\left\{n_{i}^{\prime}(t) \sqrt{2} \cos \left(\omega_{i} t+\tilde{\theta}_{i}\right)\right\},
$$

where $n_{i}^{\prime}(t)$ represents $n(t)$ after passing through the band pass filter in Fig. 2, and $L P F\{$.$\} represents low pass fil-$ tering operation. The output of the $i^{t h}$ branch correlator, $Z_{i}$, can be written as

$$
Z_{i}=S_{Z i}+N_{Z i}
$$

where

$$
\begin{aligned}
S_{Z i} & =\sum_{m=0}^{N-1} c_{m} S_{y i}\left(m M T_{c}\right), \\
N_{Z i} & =\sum_{m=0}^{N-1} c_{m} N_{y i}\left(m M T_{c}\right) .
\end{aligned}
$$


Note that signal term $S_{Z i}$ contributes to the the mean of $Z_{i}$, and the noise term $N_{Z i}$ contributes to the variance of $Z_{i}$. Consequently, the mean of $Z_{i}$, conditioned on $\alpha_{i}, \triangle_{i}$ and $d_{q}$, is given by

$$
\begin{gathered}
E\left[Z_{i} \mid \alpha_{i}, \triangle_{i}, d_{q}\right]=\sqrt{E_{c}} \alpha_{i} \cos \left(\triangle_{i}\right) \\
\cdot \sum_{m=0}^{N-1} \sum_{n=-\infty}^{\infty} d_{q} c_{n} c_{m} x\left[(m-n) M T_{c}\right] \\
= \pm N \sqrt{E_{c}} \alpha_{i} \cos \left(\triangle_{i}\right) .
\end{gathered}
$$

In the above, we have applied $x\left[(m-n) M T_{c}\right]=0$ for $m \neq n$ since $|H(f)|^{2}$ satisfies the Nyquist criterion. The variance of $Z_{i}$ is given by the variance of $N_{Z i}$ so that

$$
\operatorname{Var}\left\{Z_{i} \mid \alpha_{i}, \triangle_{i}\right\}=\operatorname{Var}\left\{N_{Z i}\right\}=N \eta_{0} / 2=\sigma^{2} .
$$

Now, combine the outputs from all the $M$ correlators to form the overall test statistic $Z$ as

$$
Z=\sum_{i=1}^{M} g_{i} Z_{i},
$$

where $g_{i}$ is the weight with which the $i^{t h}$ correlator output is multiplied before combining. For maximal ratio combining, we set [7]

$$
g_{i}=\frac{E\left\{Z_{i} \mid \alpha_{i}, \triangle_{i}\right\}}{\operatorname{Var}\left\{Z_{i} \mid \alpha_{i}, \triangle_{i}\right\}}
$$

The signal-to-noise ratio, $\rho$, at the output of the combiner can be written as

$$
\begin{aligned}
\rho & =\frac{E^{2}(Z \mid \underline{\alpha}, \underline{\triangle})}{\operatorname{Var}(Z \mid \underline{\alpha}, \underline{\triangle})} \\
& =\frac{N^{2} E_{c}}{\sigma^{2}} \sum_{i=1}^{M} \alpha_{i}^{2} \cos ^{2}\left(\triangle_{i}\right) .
\end{aligned}
$$

The conditional bit error probability is then given by

$$
p_{b}(\underline{\alpha}, \underline{\triangle})=\Phi(-\sqrt{\rho}),
$$

where

$$
\Phi(x)=\frac{1}{\sqrt{2 \pi}} \int_{-\infty}^{x} e^{-t^{2} / 2} d t .
$$

The average probability of error $p_{b}$ can be obtained by unconditioning on $\underline{\alpha}$ and $\triangle$, where $\alpha$ follows Rayleigh distribution and $\triangle$ depends on the loop SNR $\gamma$ as per Eqns. (4) and (5). For the case of $M=2$, the above unconditioning involves numerically integrating the following expression

$$
\begin{aligned}
p_{b}= & \int_{\triangle_{2}=-\pi}^{\pi} p\left(\triangle_{2}\right) d \triangle_{2} \int_{\triangle_{1}=-\pi}^{\pi} p\left(\triangle_{1}\right) d \triangle_{1}(19) \\
& \cdot \int_{\alpha_{2}=0}^{\infty} p\left(\alpha_{2}\right) d \alpha_{2} \int_{\alpha_{1}=0}^{\infty} \Phi(-\sqrt{\rho}) p\left(\alpha_{1}\right) d \alpha_{1} .
\end{aligned}
$$

As such, the evaluation of the above expression is computationally intensive. Hence, we resort to deriving a simple, inexpensive, yet reasonably accurate, bound on the average probability of error in the following sub-section.

\subsection{Derivation of upper bound on BER}

In order to make a crucial approximation in deriving a bound on the bit error probability, we make the assumption that the loop SNRs in all the $M$ PLLs are quite large, which implies that the phase estimates conditioned on the instantaneous loop SNR $\gamma$ have small variances ${ }^{2}$. When this condition holds, we may replace $\cos \left(\triangle_{i}\right)$ by its excpected value $E_{\triangle}\left[\cos \left(\triangle_{i}\right)\right]$, where $E_{\triangle}$ denotes the expectation with respect to phase error $\triangle$. From Eqn. (4)

$$
\begin{aligned}
E_{\triangle}\left[\cos \left(\triangle_{i}\right)\right] & =\int_{0}^{2 \pi} \cos \left(\triangle_{i}\right) e^{\frac{\gamma_{i} \cos \left(\triangle_{i}\right)}{2 \pi I_{0}\left(\gamma_{i}\right)}} d \triangle_{i} \\
& =\frac{I_{1}\left(\gamma_{i}\right)}{I_{0}\left(\gamma_{i}\right)} .
\end{aligned}
$$

Note that the factor $E_{\triangle}\left[\cos \left(\triangle_{i}\right)\right]=\frac{I_{1}\left(\gamma_{i}\right)}{I_{0}\left(\gamma_{i}\right)} \equiv I\left(\gamma_{i}\right)$ accounts for the degradation due to phase error compared to perfect coherence case. Thus, $I\left(\gamma_{i}\right)$ can be viewed as a phase error loss factor. In order to simplify the analysis, we approximate this phase error loss function $I($. by a staircase function having $N_{s}$ steps. That is, $I(\gamma)$ is bounded by the following function $I^{*}(\gamma)$

$$
I^{*}(\gamma)= \begin{cases}0, & \gamma \leq \beta_{N_{s}} \\ I\left(\beta_{N_{s}}\right), & \beta_{N_{s}}<\gamma \leq \beta_{N_{s}-1} \\ I\left(\beta_{N_{s}-1}\right), & \beta_{N_{s}-1}<\gamma \leq \beta_{N_{s}-2} \\ & \cdot \\ & \cdot \\ I\left(\beta_{2}\right), & \beta_{2}<\gamma \leq \beta_{1} \\ I\left(\beta_{1}\right), & \beta_{1}<\gamma<\infty,\end{cases}
$$

such that $\beta_{1}>\beta_{2}>\ldots>\beta_{N_{s}-1}>\beta_{N_{s}}>0$. Fig. 3 illustrates the true $I(\gamma)$ function and the approximating staircase function $I^{*}(\gamma)$ for $N_{s}=3$. Since the bit error probability is monotonically decreasing with $I(\gamma)$, replacing $I(\gamma)$ with $I^{*}(\gamma)$ in the subsequent analysis results in an upper bound on the bit error probability.

For $N_{s}$ steps in the approximating function, there are $N_{s}+1$ SNR range bins, viz., $\left\{0, \beta_{N_{s}}\right\},\left\{\beta_{N_{s}}, \beta_{N_{s}-1}\right\}, \ldots$, $\left\{\beta_{2}, \beta_{1}\right\},\left\{\beta_{1}, \infty\right\}$. Represent the SNR thresholds using the vector $\beta=\left[\beta_{N_{s}}, \beta_{N_{s}-1}, \ldots, \beta_{2}, \beta_{1}\right]$. Let $n_{0}$ loops fall in the SNR range $\left\{0, \beta_{N_{s}}\right\}, n_{1}$ loops fall in the SNR range $\left\{\beta_{N_{s}}, \beta_{N_{s}-1}\right\}, n_{2}$ loops fall in the SNR range $\left\{\beta_{N_{s}-1}, \beta_{N_{s}-2}\right\}$, and so on, such that $\sum_{i=0}^{N_{s}} n_{i}=M$. The vector $\mathbf{n}=\left[n_{0}, n_{1}, \ldots, n_{N_{s}}\right]$ subject to the condition $\sum_{i=0}^{N_{s}} n_{i}=M$ then represents a particular combination of the $M$ loops falling in various SNR ranges as described

\footnotetext{
${ }^{2}$ The conditional variance of $\cos \left(\triangle_{i}\right)$ has been shown to decrease quickly with increasing $\gamma_{i}$ in [5]. For $\gamma_{i} \geq 10 \mathrm{~dB} \operatorname{var}\left[\cos \left(\triangle_{i}\right)\right]$ takes values which are very small fractions of unity.
} 


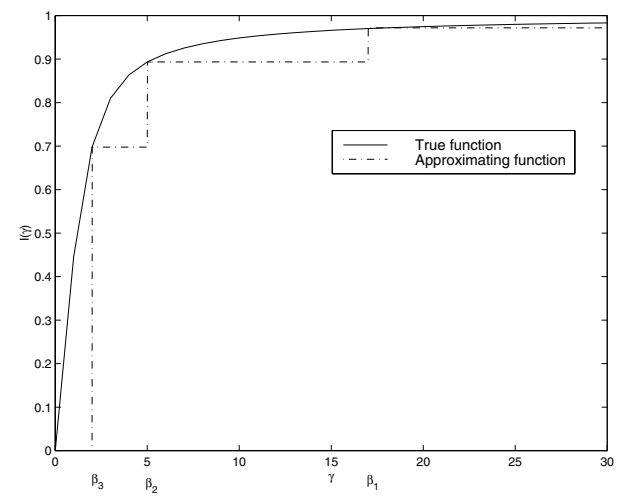

Figure 3: Approximation of the phase error loss function

above. To evaluate the average bit error probability, we carry out the following steps.

- Find the probabilities of all the possible combinations of the occurrence of the vector $\mathbf{n}$.

- For each of the above combination vector, determine the conditional bit error probability.

- Find the average bit error probability from the these conditional probabilities. This results in a family of upper bounds on the bit error probability, different for different choice of the threshold vector $\beta$.

- Choose the minimum upper bound from this family of upper bounds as the optimum upper bound on the average bit error probability.

In the following, we consider the case where $M=2$ and $N_{s}=2$ (a similar procedure applies to other values of $M$ and $\left.N_{s}\right)$. Let $P_{n 0, n 1, n 2}\left(\beta_{2}, \beta_{1}\right)$ represent the probability that $n_{0}, n_{1}, n_{2}$ loops fall in the SNR range $\left\{0, \beta_{2}\right\},\left\{\beta_{2}, \beta_{1}\right\},\left\{\beta_{1}, \infty\right\}$, respectively. The expression for $P_{0,0,2}\left(\beta_{2}, \beta_{1}\right)$ can be written as

$$
P_{0,0,2}\left(\beta_{2}, \beta_{1}\right)=\prod_{i=0}^{M-1} \int_{\beta_{1}}^{\infty} p\left(\gamma_{i}\right) d \gamma_{i}=e^{-2 \beta_{1} / \nu} .
$$

Substituting the approximation for $\cos \left(\triangle_{i}\right)$ from Eqn. (20) in Eqn. (16), the signal-to-noise ratio, $\rho^{\prime}$, at the output of the combiner can be written as

$$
\rho^{\prime}=\frac{N^{2} E_{c} I^{2}\left(\beta_{1}\right) S}{\sigma^{2}},
$$

where $S=\sum_{i=1}^{M} \alpha_{i}^{2}, \sigma_{1}^{2}=\sigma_{2}^{2}=\ldots=\sigma_{M}^{2}=\sigma^{2}$. The conditional bit error probability for this case is given by

$$
P_{b, 0,0,2}\left(\beta_{2}, \beta_{1}, S\right)=\Phi\left(-\sqrt{\rho^{\prime}}\right),
$$

where $\rho^{\prime}=\lambda S$. The pdf of $S$ is difficult to obtain because of the restrictions $\left\{\gamma_{i}\right\}>\beta_{1}$. However, by removing the restrictions on $\left\{\gamma_{i}\right\}$ (i.e., take the region $\gamma_{i}>0$ instead of $\gamma_{i}>\beta_{1}$, which gives an approximate pdf of $S)$, the resulting $S^{*}$ has a well known distribution and the upper bound remains intact because removing the restrictions is disadvantageous to the receiver and that can only increase the bit error probability. Since the $\left\{\alpha_{i}\right\}$ are Rayleigh distributed, $S^{*}$ is chi-square distributed with $2 M$ degrees of freedom, and the pdf given by $p\left(S^{*}\right)=$ $\frac{1}{\Gamma(M)} S^{*(M-1)} e^{-S^{*}}$, where $\Gamma($.$) is the Gamma function$ [7] and $M=2$. Now, averaging the conditional bit error probability expression in Eqn. (24) with respect to $S^{*}$ we get

$$
P_{b, 0,0,2}\left(\beta_{2}, \beta_{1}\right)=\int_{0}^{\infty} \Phi\left(-\sqrt{\lambda S^{*}}\right) \frac{S^{*(M-1)}}{\Gamma(M)} e^{-S^{*}} d S^{*} .(25)
$$

The above integral can be derived to be [8]

$$
P_{b, 0,0,2}\left(\beta_{2}, \beta_{1}\right)=\frac{1}{2}\left[1-\mu_{1} \sum_{k=0}^{M-1}\left(\begin{array}{c}
2 k \\
k
\end{array}\right)\left(\frac{1-\mu_{1}^{2}}{4}\right)^{k}\right]
$$

where $\mu_{1}=\sqrt{\frac{\lambda_{1}}{1+\lambda_{1}}}$ and $\lambda_{1}=\frac{N^{2} E_{c} I^{2}\left(\beta_{1}\right)}{2 \sigma^{2}}$. The expressions for $P_{b, 1,0,1}\left(\beta_{2}, \beta_{1}\right), P_{b, 0,1,1}\left(\beta_{2}, \beta_{1}\right)$, $P_{b, 0,2,0}\left(\beta_{2}, \beta_{1}\right), P_{b, 1,1,0}\left(\beta_{2}, \beta_{1}\right), P_{b, 2,0,0}\left(\beta_{2}, \beta_{1}\right)$ can be derived in a similar way.

We arrive at a family of upper bounds on bit error probability by averaging over the vector $\underline{\beta}$ as follows

$$
\begin{gathered}
P_{b}\left(\beta_{1}, \beta_{2}\right)=P_{0,0,2}\left(\beta_{2}, \beta_{1}\right) P_{b, 0,0,2}\left(\beta_{2}, \beta_{1}\right) \\
+P_{1,0,1}\left(\beta_{2}, \beta_{1}\right) P_{b, 1,0,1}\left(\beta_{2}, \beta_{1}\right)+P_{0,1,1}\left(\beta_{2}, \beta_{1}\right) P_{b, 0,1,1}\left(\beta_{2}, \beta_{1}\right) \\
+P_{0,2,0}\left(\beta_{2}, \beta_{1}\right) P_{b, 0,2,0}\left(\beta_{2}, \beta_{1}\right)+P_{1,1,0}\left(\beta_{2}, \beta_{1}\right) P_{b, 1,1,0}\left(\beta_{2}, \beta_{1}\right) \\
+P_{2,0,0}\left(\beta_{2}, \beta_{1}\right) P_{b, 2,0,0}\left(\beta_{2}, \beta_{1}\right) .
\end{gathered}
$$

The optimum upper bound is then obtained by choosing the minimum value among the family of upper bounds given by the above equation, i.e.,

$$
P_{b}^{*}=\min \left[P_{b}\left(\beta_{1}, \beta_{2}\right), \beta_{1} \geq \beta_{2} \geq 0\right] .
$$

Note that in deriving the above upper bound two major approximations were made. The first approximation involved approximating the phase error loss function by a staircase function with finite number of steps. The second approximation involved approximating the pdf of $S$ by the pdf of $S^{*}$. As we will see in the next section, these approximations do not compromise much on the accuracy of the bounds. In addition, the computational complexity involved in evaluating the bound in Eqn. (28) is quite inexpensive.

\section{Numerical results}

We evaluate the bit error performance of coherent multicarrier DS-SS systems with imperfect carrier phase on fading channels using the bounding technique and compare it with the performance in the perfect coherence case. We also establish the tightness of the bound for different values of $N_{s}$ in the approximating function by comparing with the probability of error obtained through the exact expression.

Fig. 4 shows the bit error performance as a function of $E_{b} / N_{o}\left(=M N E_{c} / N_{o}\right)$ for a system with $M=2$ and $N=256$. The loop SNR is taken to be $10 \mathrm{~dB}$ above the 
system $E_{b} / N_{o}$. Fig. 4 illustrates the performance plots for a) perfect coherence case, $b$ ) imperfect carrier phase case, computed using the exact expression in Eqn. (19), and c) imperfect carrier phase case, obtained using the bounding technique as per Eqn. (28) for $N_{s}=1,2$. The following observations can be made from Fig. 4. For the system parameters considered, $a$ ) because of imperfect phase estimates at the receiver, there is a performance loss of about $0.5 \mathrm{~dB}$ compared to the perfect coherence case, and $b$ ) the bound is loose for $N_{s}=1$ (about $2.5 \mathrm{~dB}$ above the exact value). However, when $N_{s}$ is increased to $N_{s}=2$, the bound becomes tighter (close to the exact value to within a $0.2 \mathrm{~dB}$ ). This illustrates the accuracy and simplicity of the bound.

Next, the effect of loop SNR on the performance of the system with imperfect carrier phase in comparsion with that of a perfect coherence system for $M=2, N=256$ and $N_{s}=2$ is shown in Fig. 5. It is observed that, as expected, the performance loss due to imperfect carrier phase becomes increasingly less as the loop SNR is increased. For example, when the loop SNR is $20 \mathrm{~dB}$ above the system $E_{b} / N_{o}$, the performance loss is only less than 0.25 dB. Finally, Fig. 6 illustrates the effect of increasing the number of sub-carriers (to $M=3$ ) on the performance when $N=256, N_{s}=2$ and loop SNR is $10 \mathrm{~dB}$ above $E_{b} / N_{o}$. As the number of sub-carriers is increased, the performance improves indicating the robustness of the multicarrier system to multipath fading.

\section{Conclusions}

We analyzed the bit error performance of multicarrier DS-SS systems with imperfect carrier phase on multipath Rayleigh fading channels. We considered the phase errors arising from the receiver phase locked loops on individual sub-carriers to be Tikhonov distributed. We derived a simple, approximate upper bound on the average bit error probability. We showed that the bound is inexpensive to compute and is reasonably accurate. We also showed that, compared to the perfect coherence case, the bit error performance in the case of imperfect carrier phase degrades by less than $1.5 \mathrm{~dB}$ and $0.25 \mathrm{~dB}$ for loop SNR values of $10 \mathrm{~dB}$ and $20 \mathrm{~dB}$ above the system $E_{b} / N_{o}$, respectively.

\section{References}

[1] N. Yee, J. P. Linnartz, and G. Fettweis, "Multicarrier CDMA in indoor wireless radio," Proc. PIMRC'93, pp. D1.3.1-5, December 1993.

[2] S. Kondo and L. B. Milstein, "Performance of multicarrier DSCDMA systems," IEEE Trans. Commun., vol. 44, no.2, pp.238246, February 1996.

[3] E. A. Sourour and M. Nakagawa, "Performance of orthogonal multicarrier CDMA in a multipath fading channel," IEEE Trans. Commun., vol. 44, no.3, pp.356-367, March 1996.

[4] W. Xu and L. B. Milstein, "Performance of multicarrier DS CDMA systems in the presence of correlated fading," Proc. VTC'97, pp.2050-2054, 1997.

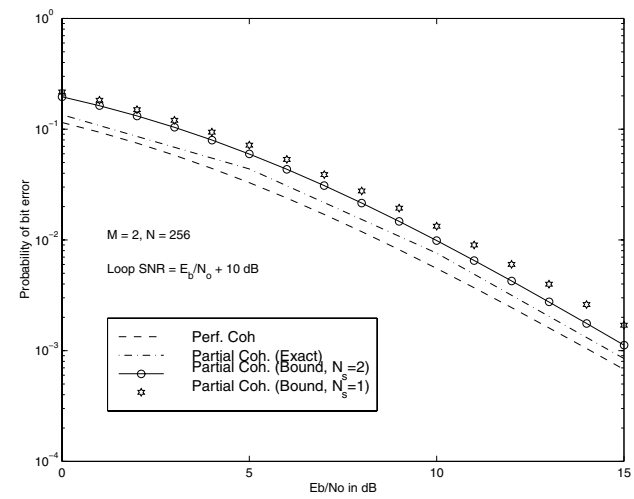

Figure 4: Bit error probability bounds with imperfect carrier phase in Rayleigh fading. $M=2, N=256$.

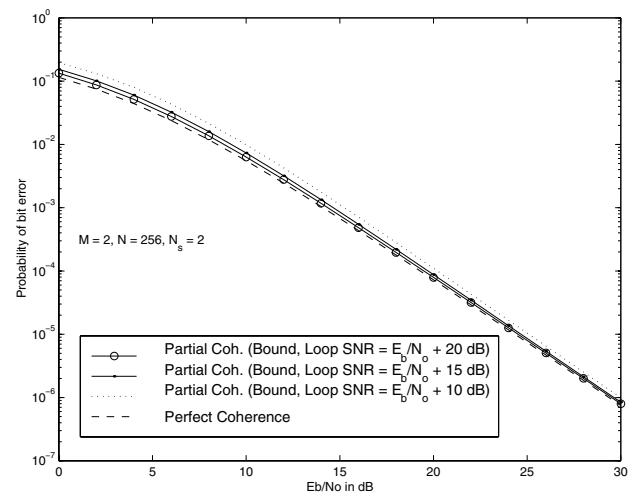

Figure 5: Bit error probability bounds with imperfect carrier phase different loop SNR. Rayleigh fading. $M=2, N=256, N_{s}=2$.

[5] T. Eng and L. B. Milstein, "Partially coherent DS-SS performance in frequency selective multipath fading," IEEE Trans. Commun., vol. 45, no.1, pp.110-118, January 1997.

[6] A. J. Viterbi, Principles of coherent communication, McGrawHill, 1966.

[7] J. G. Proakis, Digital Communications, McGraw-Hill, 1995.

[8] T. Eng and L. B. Milstein, "Coherent DS-CDMA performance in Nakagami multipath fading," IEEE Trans. Commun., vol. 43, pp.1134-1143, March 1995.

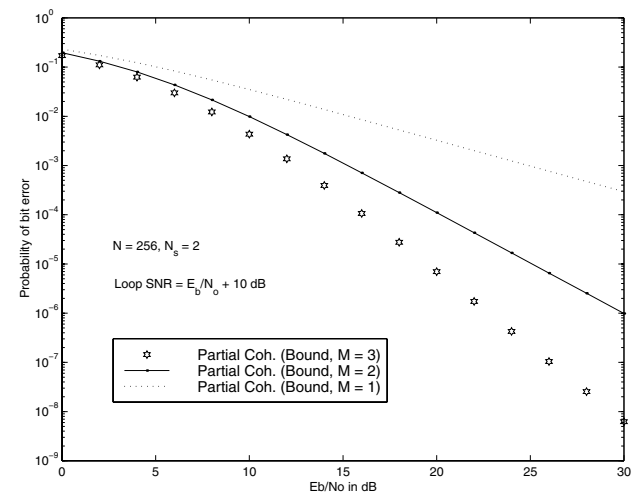

Figure 6: Bit error probability bounds with imperfect carrier phase as a function of number of sub-carriers $M$. Rayleigh fading. $M=2,3$, $N=256, N_{s}=2$. 